\title{
First Demonstration of Ionization Cooling by the Muon Ionization Cooling Experiment
}

\section{J. Pasternak ${ }^{a, b, 1, *}$}

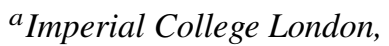
Exhibition Road, London, SW7 2AZ, UK

${ }^{b}$ STFC Rutherford Appleton Laboratory, Harwell Oxford, Didcot, OX11 OQX, UK

E-mail: j.pasternak@imperial.ac.uk

Muon colliders have the potential to carry the search for new phenomena to energies well beyond the reach of the LHC in the same or smaller footprint. Muon beams may be created through the decay of pions produced in the interaction of a proton beam with a target. To produce a high-brightness beam from such a source requires that the beam be cooled. Ionization cooling is the novel technique by which it is proposed to cool the beam. The Muon Ionization Cooling Experiment collaboration has constructed a section of an ionization cooling cell and used it to provide the first demonstration of ionization cooling. Here the observation of ionization cooling is described. The cooling performance is studied for a variety of beam and magnetic field configurations. The cooling performance is related to the performance of a possible future muon collider facility.

40th International Conference on High Energy physics - ICHEP2020

July 28 - August 6, 2020

Prague, Czech Republic (virtual meeting)

${ }^{1}$ For the MICE Collaboration.

* Speaker 


\section{Introduction}

Muons are considered excellent beam particles for collider applications due to their unique properties. As they are elementary leptons, a large QCD background known in hadron colliders can be avoided and the full $\mu^{+} \mu^{-}$centre of mass energy of the collision can be used for creation of new particle states. Due to a relatively large muon mass, $\sim 200$ times larger than electron, the synchrotron radiation is suppressed, which offers a unique opportunity to use compact rings for beam acceleration and storage, and to avoid beam quality degradation at high energy due to beamstrahlung. Furthermore, the large muon mass offers an increased coupling to the Higgs boson compared to the electron case and provides a possibility to explore the resonant s-channel Higgs production. A multi-TeV Muon Collider has a physics reach comparable with $100 \mathrm{TeV}$ proton-proton collider, with a footprint compatible with existing laboratories [1].

Over the years a very significant effort was devoted to the design of the Muon Collider and solving problems necessary to demonstrate the feasibility of its subsystems. Several schemes were proposed to produce muon beams suitable for collider applications [2], among which a proton-based production scheme has been studied in significant details. In this scheme, protons incident on a target produce pions which decay into muons. The resulting muon beam has a very large initial emittance and energy spread, which requires a significant beam cooling in order to be able to achieve a sufficient luminosity in the collider applications. Due to the shortness of the muon lifetime the only cooling technique fast enough to be applicable to the muon beams is ionization cooling [3-5], where muons are passing through an absorber material immersed in a strongly focusing lattice losing total momentum via ionization and subsequently recovering the longitudinal component of momentum only in RF re-acceleration. The ionization cooling of muons has been demonstrated for the first time experimentally by the Muon Ionization Cooling Experiment (MICE) at RAL [6].

In this paper, after describing the MICE experiment, the current status of ionization cooling analysis is discussed.

\section{MICE experiment}

The MICE experiment used a muon beam produced at a titanium target dipped in the edge of the proton beam in the ISIS synchrotron at RAL [7]. Pions produced at the target were captured in a beam line consisting of quadrupoles, dipoles and a decay solenoid, where the muon beam was formed and transported to the MICE cooling channel [8]. The MICE cooling channel consisted of twelve superconducting solenoids subdivided into three magnet modules. The central Focused Coil module contained the absorber. Two coils, which could be operated with the same or opposite polarity, provided strong beam focusing required to achieve ionization cooling. Two identical modules placed anti-symmetrically upstream and downstream of the Focus Coil (Spectrometer Solenoids) were made out of five coils each. Three were grouped together to provide the uniform magnetic field for the Tracker detectors [9] and two others were used to perform beam matching into and out of the Focus Coil module. At the entrance to the upstream Spectrometer Solenoid module a variable thickness brass and tungsten diffuser was placed, which allowed to vary the incident beam emittance. The MICE beam line was instrumented with several sub-detectors both upstream and downstream of the absorber [10]. The Time Of Flight (TOF) detectors together with threshold 
Cherenkov counters (Ckov A and Ckov B) were used for particle velocity measurements. Two scintillating fibre Trackers measured particle position and momentum before and after passing the absorber module. Downstream of the cooling channel an additional TOF station, a mixed lead and scintillator pre-shower detector (KL), and a totally active scintillator calorimeter, the Electron Muon Ranger (EMR) were used to cross-validate the measurements of upstream detectors and identify electrons from the muon decay. The MICE detector system was designed to measure particle position, momentum, velocity, energy and particle ID on a particle-by-particle basis, which was essential to perform the world's first single particle emittance measurement [11]. The schematic of the MICE apparatus with its subsystems is shown in figure 1.

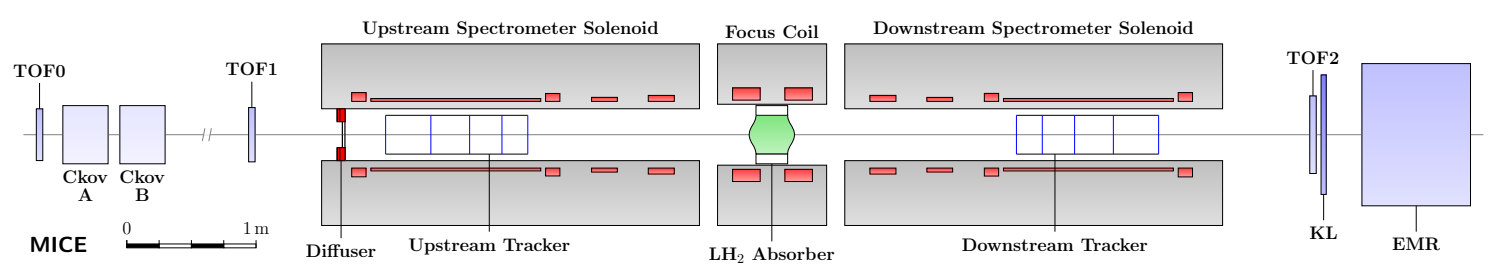

Figure 1: Schematic of the MICE apparatus with its subsystems.

\section{MICE analysis}

The main goal of the MICE experiment is to demonstrate ionization cooling of muons using low-Z absorbers, which allows to minimise the heating effect from the multiple Coulomb scattering. In particular, liquid hydrogen ('Full $\mathrm{LH}_{2}$ ') and lithium hydride (' $\mathrm{LiH}$ ') absorbers were tested. For comparison, data were also taken with the evacuated $\mathrm{LH}_{2}$ absorber ('Empty $\mathrm{LH}_{2}$ ') and with the absorber module completely removed ('No Absorber'). MICE was operated in two main magnetic configurations: in the solenoid mode, where the polarity of the magnetic field was positive upstream and downstream of the Focus Coil module, and in the flip mode, with the polarity changing across the Focus Coil. Due to an accident which occurred during the commissioning, one of the matching coils in the downstream Spectrometer Solenoid was inoperable. This required re-optimization of the optics in the cooling channel, in which a compromise between the strength of focusing at the absorber and the beam transmission was necessary. The final magnetic configurations and resultant beam envelopes for beams with various initial emittances and the reference momentum of $140 \mathrm{MeV} / \mathrm{c}$ are shown in figure 2.

After selecting the sample within the momentum window of 135-145 MeV/c, events were required to pass the quality test for the track reconstruction in both Trackers [12] and be fully contained in the fiducial volume. Monte Carlo simulations were extensively used to test the performance of the MICE cooling channel, and to study the resolution and efficiency of detectors. The main variable of interest for the demonstration of ionization cooling is the root-mean-square normalized transverse 4D emittance. In the absence of cooling and heating effects it is approximately conserved quantity during motion of the beam along the magnetic lattice. An equivalent and related quantity is the single particle transverse amplitude. The evolution of the distribution of single particle transverse amplitudes was used in the world's first demonstration of ionization cooling by MICE experiment [6]. In particular, a very clear sign of cooling can be seen in the downstream to 


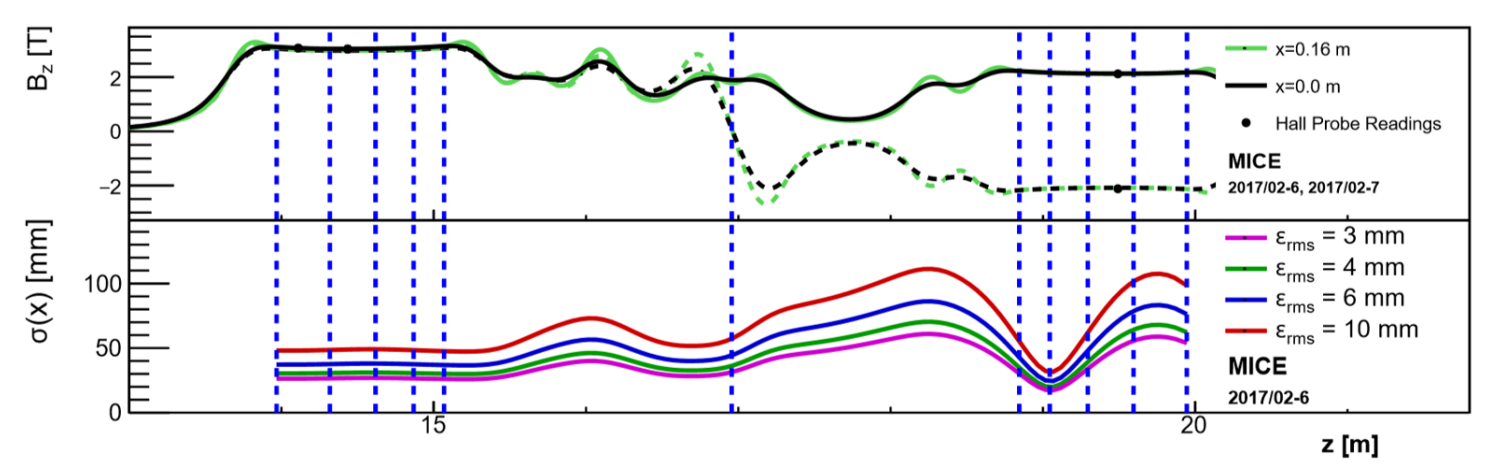

Figure 2: The calculated magnetic field, $B_{z}[\mathrm{~T}]$, in the solenoid (solid lines) and flip modes (dashed lines), and nominal horizontal width of the beam in the flip mode, $\sigma(x)[\mathrm{mm}]$. The modelled field is shown on the beam axis and $160 \mathrm{~mm}$ from the axis in the horizontal plane. The readings of Hall probes, situated $160 \mathrm{~mm}$ from the beam axis, are also shown. Dashed vertical lines indicate the position of the tracker stations and absorber. The nominal RMS beam width is calculated assuming a nominal input beam using linear beam transport equations.

upstream ratio of number of events plotted as a function of the transverse amplitude, as shown in figure 3 for the flip mode configuration. A value larger than one indicates migration of particles towards the core of the beam after passing through the absorber. This is clearly visible for the 'Full $\mathrm{LH}_{2}$ ' and ' $\mathrm{LiH}$ ' absorbers within the acceptance of the channel, and indicates the presence of cooling process. A value compatible with one is visible for the 'Empty $\mathrm{LH}_{2}$ ' and 'No Absorber' cases within the limits of the acceptance, which is expected in the absence of cooling. The collaboration continues to work towards extending this analysis to the solenoid mode [13] and to include other values of the beam momentum.

An alternative analysis is in preparation focusing on obtaining more quantitative results on the transverse normalized emittance reduction by applying a rejection sampling technique to the parent distribution reconstructed in the upstream Tracker [14]. This allows to vary the input emittance considered in the analysis, improve the beam matching to the upstream Tracker and the absorber, and enhance the transmission for the selected sample. This analysis demonstrates that, for beams with input emittances in the $1.5-5 \mathrm{~mm}$ range, the absolute change in emittance induced by the $\mathrm{LiH}$ or $\mathrm{LH}_{2}$ absorbers is negative for large emittances and positive for low emittances. This approach allows a measurement of the equilibrium emittance, where heating due to multiple Coulomb scattering balances cooling due to ionization, yielding the net zero change. This clear sign of the ionization cooling is shown in figure 4 . The 'No absorber' case is compatible with the emittance conservation, while the 'Empty $\mathrm{LH}_{2}$ ' one shows a slight heating, which can be explained by the presence of the aluminium windows. The beams are sampled from parent ensembles with nominal input emittance of $6 \mathrm{~mm}$ and nominal input momentum of $140 \mathrm{MeV} / \mathrm{c}$.

Another important area of the MICE analysis is focusing on the data obtained with the wedge absorber, which is the key element in demonstrating the longitudinal emittance evolution required for the Muon Collider. The results of Monte Carlo simulations of the MICE channel equipped with the wedge absorber are presented in reference [15]. 


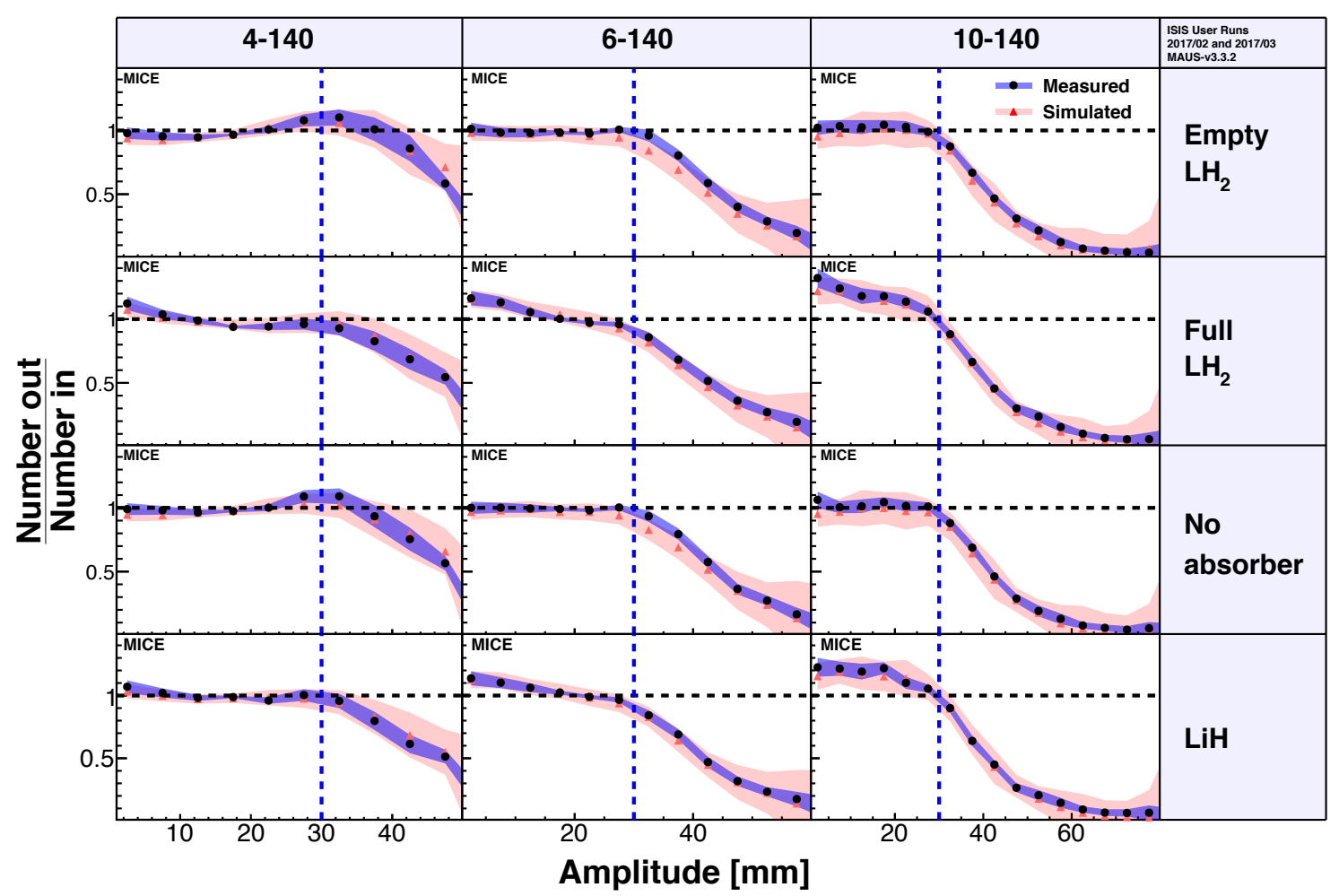

Figure 3: Downstream to upstream ratio of number of events as a function of the transverse amplitude. The effect predicted from the Monte Carlo simulation is shown in red, while that measured is shown in black. Uncertainty is shown by a blue fill for data and a pink fill for simulation and is dominated by systematic uncertainty. Vertical lines indicate the channel acceptance above which scraping occurs. The results are shown for three samples with different input emittances of 4, 6 and $10 \mathrm{~mm}$ at $140 \mathrm{MeV} / \mathrm{c}$ (denoted as '4-140', '6-140' and '10-140', respectively). A value greater than one is a clear sign of the ionization cooling.
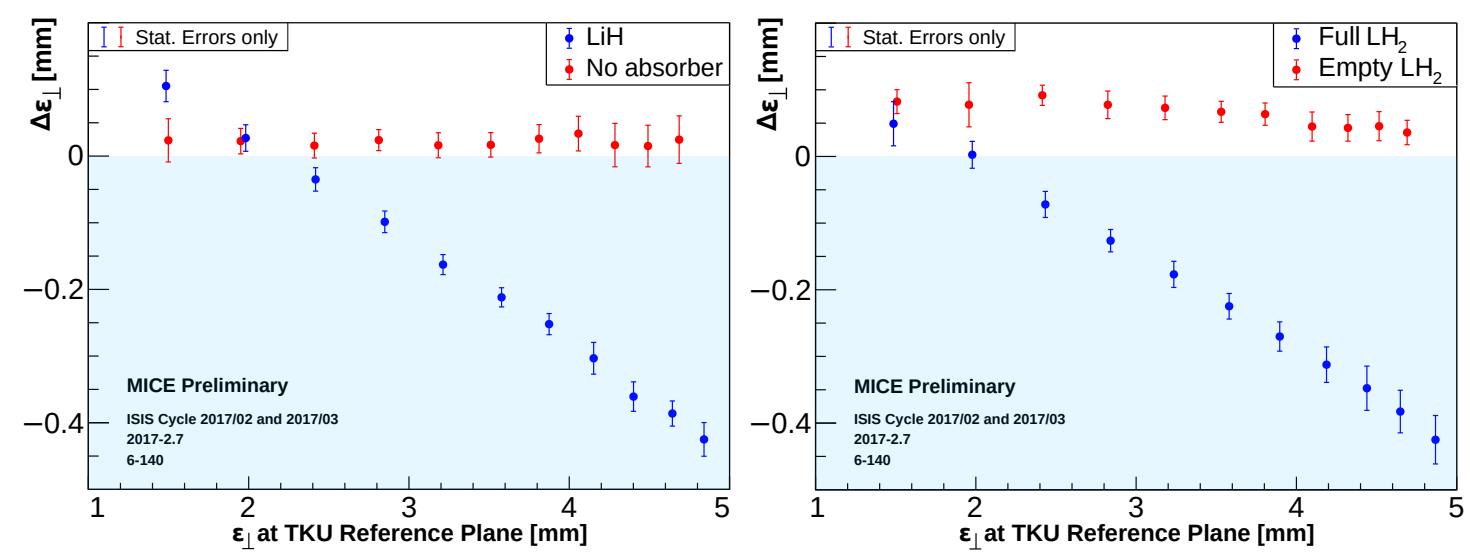

Figure 4: Absolute emittance change between upstream and downstream tracker reference planes as a function of beam emittance at the upstream Tracker. Comparisons between (left) 'LiH' and 'No absorber' data, and (right) 'Full $\mathrm{LH}_{2}$ ' and 'Empty $\mathrm{LH}_{2}$ ' data indicate cooling in the presence of an ionizing material of the absorbers. The error bars represent the values of statistical errors. 


\section{Conclusions}

MICE demonstrated experimentally the principle of ionization cooling for muon beams [6] opening the route for the development of future muon accelerators like the Neutrino Factory or a Muon Collider. The collaboration continues to explore the data collected to obtain further insight into the physics of ionization cooling.

\section{References}

[1] J. P. Delahaye, M. Diemoz, K. Long, B. Mansoulié, N. Pastrone, L. Rivkin, D. Schulte, A. Skrinsky, and A. Wulzer, Muon colliders (2019), arXiv:1901.06150 [physics.acc-ph] .

[2] M. Boscolo, J.-P. Delahaye, and M. Palmer, Rev. Accel. Sci. Tech. 10, 189 (2019), arXiv:1808.01858 [physics.acc-ph] .

[3] A. Skrinsky and V. Parkhomchuk, Sov. J. Part. Nucl. 12, 223 (1981).

[4] D. Neuffer, Conf. Proc. C 830811, 481 (1983).

[5] D. Neuffer, Part. Accel. 14, 75 (1983).

[6] M. Bogomilov et al. (MICE), Nature 578, 53 (2020), arXiv:1907.08562 [physics.acc-ph] .

[7] C. Booth et al., JINST 8, P03006, arXiv:1211.6343 [physics.ins-det] .

[8] M. Bogomilov, Y. Karadzhov, D. Kolev, I. Russinov, R. Tsenov, G. Vankova-Kirilova, L. Wang, F. Y. Xu, S. X. Zheng, R. Bertoni, and et al., Journal of Instrumentation 7 (05), P05009-P05009.

[9] M. Ellis et al., Nucl. Instrum. Meth. A 659, 136 (2011), arXiv:1005.3491 [physics.ins-det] .

[10] D. Orestano (MICE), Nucl. Instrum. Meth. A 617, 45 (2010).

[11] D. Adams et al. (MICE), Eur. Phys. J. C 79, 257 (2019), arXiv:1810.13224 [physics.acc-ph] .

[12] A. Dobbs, C. Hunt, K. Long, E. Santos, M. Uchida, P. Kyberd, C. Heidt, S. Blot, and E. Overton, Journal of Instrumentation 11 (12), T12001-T12001.

[13] T. Lord, in Proc. of 40th International Conference on High Energy physics - ICHEP2020 (2020) p. Posters / 56.

[14] P. Jurj, in Proc. of 40th International Conference on High Energy physics - ICHEP2020 (2020) p. Posters / 54.

[15] C. Brown, in Proc. of 40th International Conference on High Energy physics - ICHEP2O20 (2020) p. Posters / 55. 